\title{
Ökonomische Aspekte und systemtheoretische Denkansätze als Erklärungsmodell für die Wirkung einer komplexen medizinischen Kur
}

\section{Economical Aspects and System Theoretical Considerations as Attempted Explanation for the Effects of a Complex Medical Spa Therapy}

\author{
Autor \\ H. Schoberwalter ${ }^{1,2}$, G. Haber ${ }^{3}$, W. Marktl \\ Institut \\ ${ }^{1}$ Wiener Internationale Akademie für Ganzheitsmedizin, Österreich
2 Pensionsversicherungsanstalt Wien, Österreich
${ }^{3}$ Donau-Universität Krems, Österreich
}

Schlüsselwörter

- Komplexe Kur

- Ökonomische Aspekte

- Systemtheorie

Key words

- complex spa therapy

- economical aspects

system theory

eingereicht: $\quad 23.06 .2015$

angenommen: 10.12 .2015

\section{Bibliografie}

DOI http://dx.doi.org/ 10.1055/s-0041-111517

Phys Med Rehab Kuror 2016; 26: 59-66

(c) Georg Thieme Verlag KG

Stuttgart · New York

ISSN 0940-6689

Korrespondenzadresse

Senator MR Dr.

\section{H. Schoberwalter}

Wiener Internationale

Akademie für Ganzheitsmedizin

Sanatoriumstraße 2

A-1140 Wien

schoberwalter@gamed.or.at

\section{Zusammenfassung}

$\nabla$

Komplexe medizinische Kuren sind vor allem in den Ländern Mittel- und Osteuropas traditionelle gesundheitsförderliche Verfahren von nicht nur gesundheitspolitischer, sondern auch ökonomischer Bedeutung. Die ökonomische Bedeutung wird am Beispiel der Situation ambulanter und stationärer Heilverfahren in Österreich in den letzten 10 Jahren illustriert. Angesichts der Stellung der balneologischen Kur im Gesundheitswesen erscheint es aber auch notwendig die, solchen komplexen gesundheitsdienlichen Verfahren zugrunde liegenden Prinzipien, auf der Basis neuer Erkenntnisse zu diskutieren. Komplexe medizinische Verfahren, bei denen eine Mehrzahl von gesundheitsförderlichen Interventionen einer Mehrzahl von physischen und psychischen Effekten gegenüber steht, sollten nach dem Stand des derzeitigen Wissens einer systemtheoretischen Betrachtung unterzogen werden. Auf diese Weise sollte es möglich sein, den gesundheitlichen Stellenwert von Kurheilverfahren objektiv zu untermauern.

\section{Ökonomische Aspekte der komplexen medizinischen Kur \\ $\nabla$}

Komplexe medizinische Kuren können sowohl aus gesundheitspolitischer als auch aus volkswirtschaftlicher Sicht Interesse beanspruchen. Am Beispiel der Verhältnisse in Österreich kann gezeigt werden, dass das Kurwesen einen nicht zu unterschätzenden ökonomischen Faktor darstellt [1].

Die durchgeführten Heilverfahren lassen sich in solche unterscheiden, welche im Rahmen der Prävention bzw. Gesundheitsvorsorge (GV) als Heilverfahren-Kur (HV-Kur) bezeichnet werden, als auch jene im Rahmen der Rehabilitation bzw. Wiederherstellung, die daher HeilverfahrenRehabilitation (HV-Rehab) genannt werden. Wie

\section{Abstract}

$\nabla$

Complex balneologic spa therapies are traditional health promoting systems especially in several countries of middle and east Europe. They demand importance not only for health politics but also for health economy. An example for the economical importance of spa therapy will be given by the development of stationary as well as ambulant medical treatments in spas during the last 10 years in Austria. Taking into account the medical value of balneologic spa therapies in the public health system, it seems to be necessary to discuss the principles underlying such complex health promoting systems on a basis of new scientific considerations. Complex medical treatments characterized by a majority of different health promoting interventions with effects on a majority of physiological as well as psychological functions, should be, according to the present knowledge, submitted to the considerations of system theory. In this way it should be possible to corroborate in an objective manner the health value of complex spa therapy.

aus der $\bullet$ Abb. 1 hervorgeht, hat die Zahl der erledigten Anträge auf Heilverfahren an die Sozialversicherungsträger im Zeitraum von 2002 bis 2012 genauso kontinuierlich zugenommen, wie die Zahl der genehmigten Anträge und die Einweisung in eine stationäre Behandlung. Von den insgesamt über 260000 Einweisungen in stationäre Behandlung im Jahr 2012 entfiel mehr als die Hälfte auf Kuraufenthalte ( $\bullet$ Abb. 2).

Die $\odot$ Abb. 3a und $3 \mathbf{b}$ zeigt die Entwicklung der stationären Heilverfahren (HV-Kuren) im Rahmen der Gesundheitsvorsorge (GV) in Österreich in den Jahren 2005 bis 2014 - Gesamtergebnis und nach Indikationen.

Die $\bullet$ Abb. 4 definiert die Entwicklung der stationären (HV-REHAB) und ambulanten Rehabilitation in Österreich in den Jahren 2005-2014. 


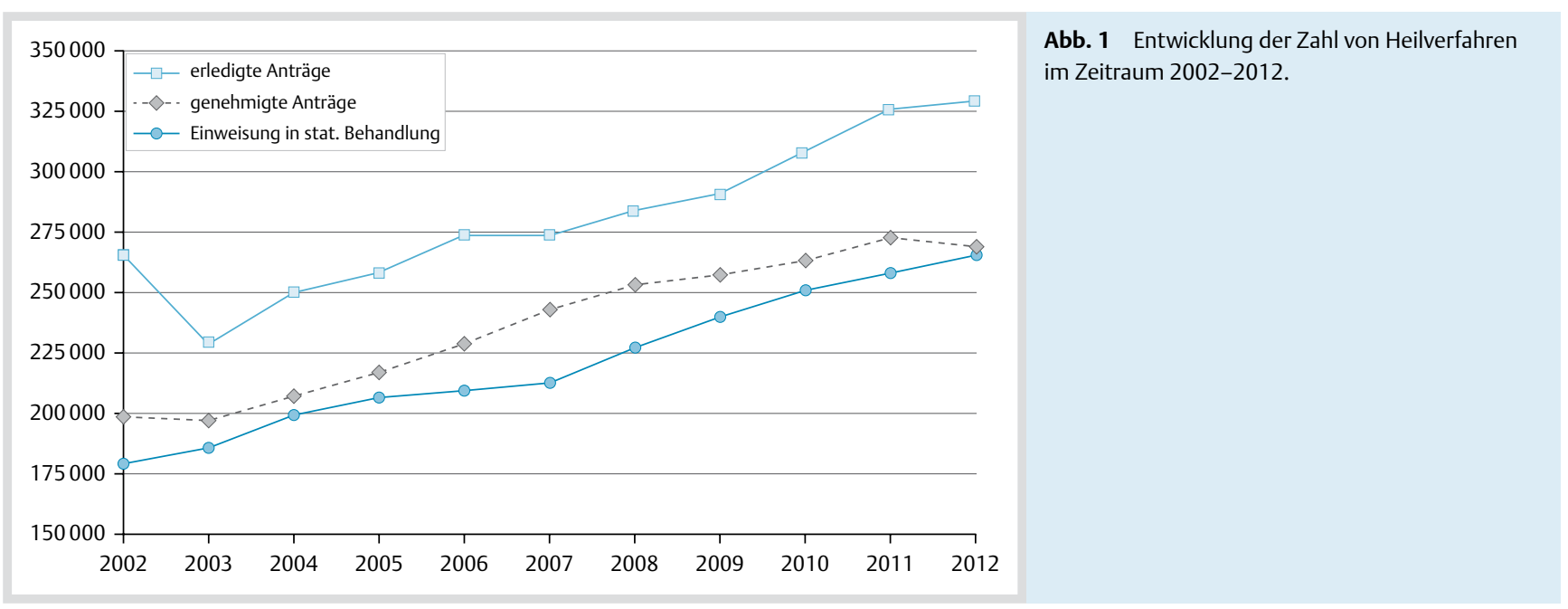

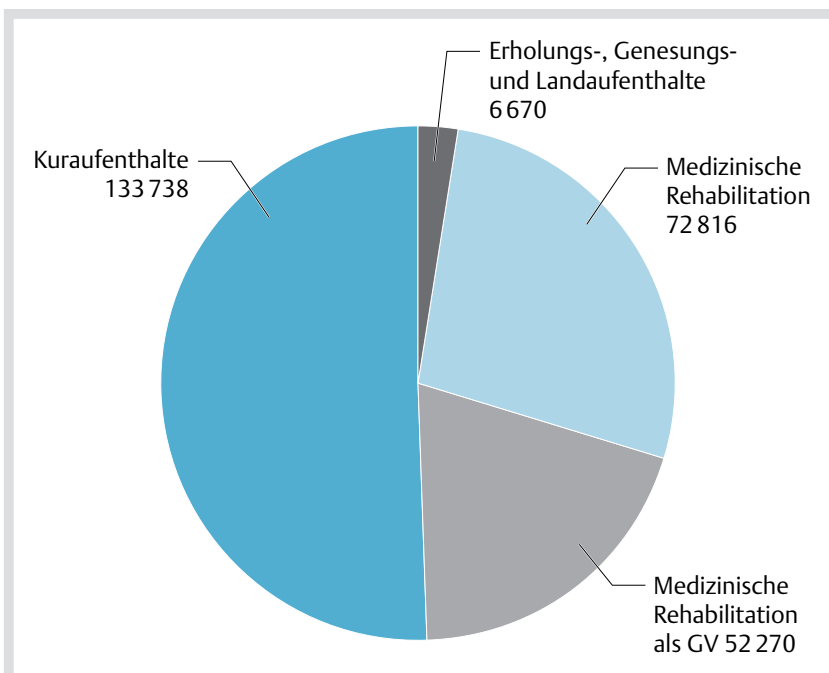

Abb. 2 Verteilung von Heilverfahren in Österreich im Jahr 2012.

Die $\triangle$ Abb. 5 präsentiert die Entwicklung der durchgeführten stationären Rehabilitation (HV-REHAB) im selben Zeitraum nach Indikationen. Die $\bullet$ Abb. 6 zeigt die Entwicklung der ambulanten Rehabilitation in den Jahren 2007-2014 ebenfalls nach Indikationen. Die Daten wurden von der Pensionsversicherung (PVA), Abteilung Statistik und Controlling (HSCO) in Wien zur Verfügung gestellt.

Die Auslastung der 116 Kureinrichtungen in Österreichs betrug im Jahr 2013 74\%. 14,3\% der Gesamtübernachtungen in Österreich betrafen im Jahr 2013 den Kurtourismus. Allerdings zeichnet sich für das Kurwesen eine Änderung der Entwicklung ab, als die Zuweisungen zu Rehabilitationsaufenthalten zulasten der Kuraufenthalte zunehmen.

Wie Haber [1] in einer Studie ausführt, stellt das Kurwesen im Rahmen der österreichischen Wirtschaftsleistung und Beschäftigung einen, im Verhältnis zur geringen absoluten Größe aufgrund der engen Abgrenzung dieses Bereichs, nicht zu unterschätzenden ökonomischen Faktor mit volkswirtschaftlicher Bedeutung dar. So beträgt der Anteil des Kurwesens an der gesamten Wertschöpfung 0,13, und 0,09\% der Arbeitsplätze sind österreichweit direkt oder indirekt auf das Kurwesen zurückzuführen.

Aus verschiedenen, nicht zuletzt ökonomischen Gründen, erlangt neben der kurativen klinischen Medizin zunehmend die Prävention (Gesundheitsvorsorge) und Rehabilitation (Wieder- herstellung) Bedeutung. Diese Entwicklung eröffnet auch der komplexen medizinischen Kur neue Chancen. Balneologische Kuren können einerseits, was die Wirkungen der natürlichen ortsgebundenen Heilvorkommen anlangt, nach naturwissenschaftlichen Kriterien beurteilt werden, die während einer Kur durchgeführten gesundheitsförderlichen Interventionen beschränken sich andererseits aber nicht nur auf die wiederholte Anwendung des natürlichen Kurmittels. Kureffekte und Kurerfolg sind das Resultat verschiedener, sogenannter unspezifischer und spezifischer Maßnahmen, die in ihren Auswirkungen nicht getrennt voneinander betrachtet werden dürfen. Ein Verständnis der gesundheitlich erwünschten Wirkung von Kuren bedarf daher nicht nur einer reduktionistisch naturwissenschaftlichen Denkungsweise, sondern muss auf dem Verständnis systemtheoretischer Grundlagen beruhen. Der medizinische Wert der komplexen balneologischen Kur kann auf dieser intellektuellen Grundlage verstanden werden und der Kur auch weiterhin ihren Stellenwert im Gesundheitssystem sichern. Dies erscheint angesichts der ökonomischen Bedeutung der Kur gerechtfertigt.

\section{Wesensmerkmale der komplexen medizinischen Kur $\nabla$}

Einleitend sei zu den in Österreich verwendeten Begriffen der Heilverfahren-Kur im Rahmen der Gesundheitsvorsorge und als Heilverfahren-Rehabilitation im Rahmen der Wiederherstellung Folgendes festzuhalten:

Maßnahmen der Gesundheitsvorsorge (Heilverfahren als Kur) dienen für den Sozialversicherungsträger Pensionsversicherung, auf deren statistische Zahlen als größter Sozialversicherungsträger Österreichs zugegriffen wurde, bei Berufstätigen der Erhaltung der Leistungsfähigkeit. Bei Pensionsbeziehern soll der Eintritt der Pflegebedürftigkeit vermieden oder der Status der Pflegebedürftigkeit verbessert werden bzw. erhalten bleiben.

Maßnahmen der Wiederherstellung (Heilverfahren als Rehabilitation) dienen der Erhaltung oder Wiedergewinnung der Fähigkeit zur Berufsausübung und zur aktiven Teilnahme am normalen Leben in Familie und Gesellschaft.

Ein stabiler Krankheitszustand und eine abgeschlossene Krankenbehandlung sind Voraussetzung für den Rehabilitationsbeginn.

Komplexe medizinische Kuren stellen eine Maßnahme der Gesundheitsförderung dar, die vor allem in Europa, in anderen Formen aber auch auf anderen Kontinenten, zu den traditionellen medizinischen Verfahren gezählt werden können. 


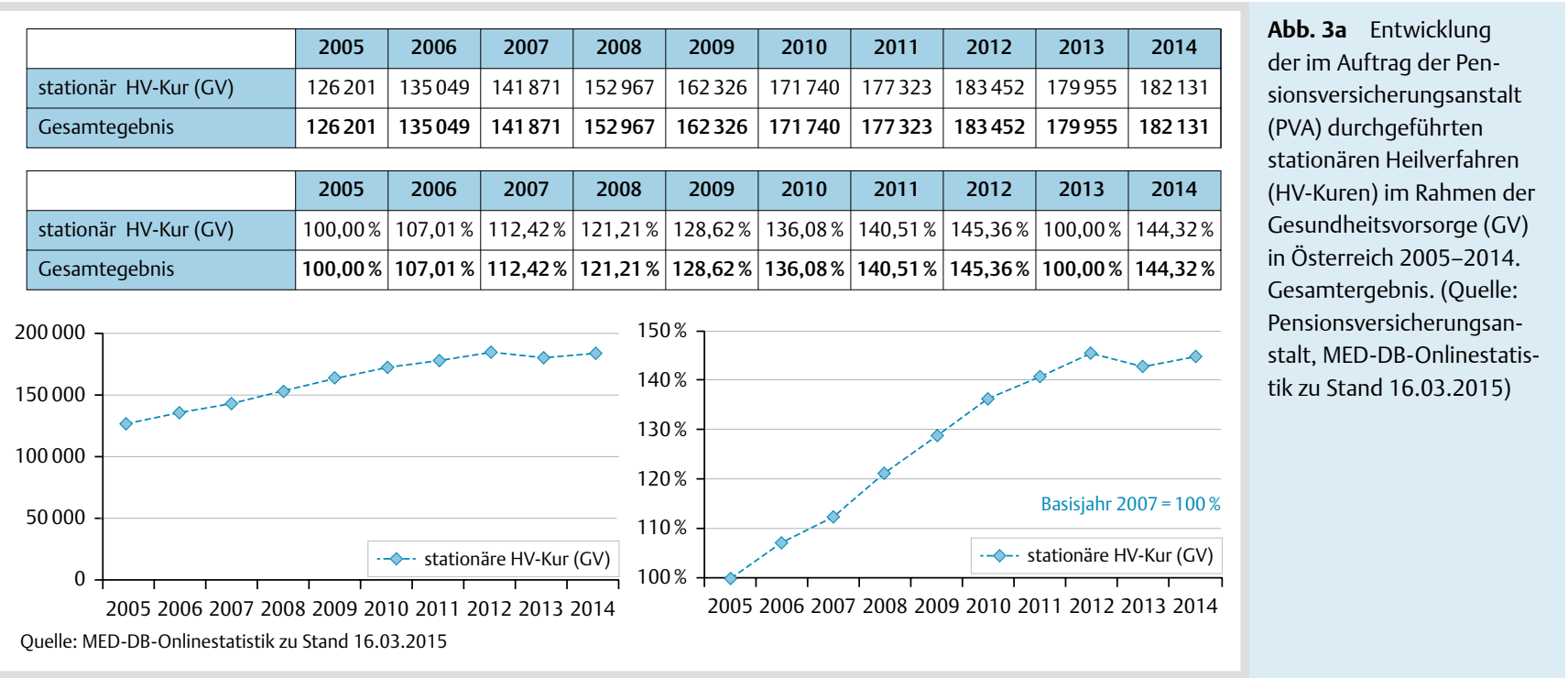

Der Begriff der Komplexität kann im Zusammenhang mit der medizinischen Kur aus verschiedenen Blickwinkeln betrachtet und definiert werden. Eine dieser Betrachtungsebenen bezieht sich auf die Mehrzahl unterschiedlicher Reize mit therapeutischer Relevanz, die während einer Kur zur Anwendung gelangen und in ihrer Gesamtheit den Kureffekt ausmachen. Dieser Kureffekt manifestiert sich dann nicht nur in einer messbaren Verbesserung einzelner Parameter und Symptome, sondern z.B. auch im Hinblick auf psychologische Faktoren wie Befinden, Schlafqualität, subjektive Stressbelastung usw. [2-6]. Einer Mehrzahl von therapeutischen Reizen steht somit eine Mehrzahl von therapeutisch erwünschten Effekten gegenüber. In der klinischen Medizin, die auf einer naturwissenschaftlichen Basis beruht, geht es nicht selten um einfache Ursache-/Wirkungsbeziehungen, wie sie dem Postulat eines experimentellen Ansatzes entsprechen. Das sich daraus ergebenden Kausalitätsprinzip fordert den Nachweis, dass eine bestimmte Ursache bzw. therapeutische Maßnahme mit dem gemessenen Effekt in einer direkten und kausalen Beziehung steht. Daraus kann abgeleitet werden, dass dieses Prinzip für komplexe Therapieverfahren und komplexe Reaktionen nicht angewendet werden kann. Aus diesem Grund ist der für die Naturwissenschaft typische reduktionistische Ansatz für eine Erklärung der komplexen Kur nicht optimal. In der Balneologie herrscht weitgehend Einstimmung darüber, dass die therapeutisch erwünschten Effekte nicht die einfache und direkte Folge der Reizeinwirkung sind, sondern auf den physiologischen Reaktionen des Organismus beruhen. Die Balneotherapie wird daher als Reiz-Reaktionstherapie bezeichnet und den regulationstherapeutischen Verfahren zugeordnet. Bei therapeutischen Verfahren dieser Art ist es nicht möglich, isoliert einzelne physiologische Regulationen zu beeinflussen, weil die physiologischen Regulationen des Organismus eine netzwerkartige Struktur [7-13] aufweisen. Sie zeigen eine wechselseitige funktionelle Abhängigkeit und weisen Charakteristika von komplexen Systemen auf [14-17]. Solche Systeme weisen auch eine Abhängigkeit von Umweltbedingungen und zeitlichen Aspekten auf. Diese Abhängigkeiten rechtfertigen in sachlicher Hinsicht die verschiedenen unspezifischen Therapiefaktoren der Kur und begründen die Zeitdauer, die für Kuren angesetzt wird. Im Zusammenhang mit der Kurdauer ist auch auf die Bedeutung adaptationsphysiologischer Vorgänge für den Kureffekt hinzuweisen, wie dies vor allem von Hildebrandt [18] betont wurde.
Alle für die Kur und ihre Wirkung relevanten exogenen und endogenen Faktoren werden auch bei systemtheoretischen Betrachtungen komplexer therapeutischer Systeme wie der TCM oder Ayurveda als Modell der Erklärung ihrer Effektivität herangezogen. Dabei wird jedoch wenig berücksichtigt, dass gerade bei komplexen gesundheitsförderlichen Systemen der kulturelle Aspekt eine nicht unerhebliche Rolle spielen kann. Ein gesundheitsförderliches Verfahren wie es die komplexe balneologische Kur darstellt, steht jedoch auf dem Boden der Tradition in den europäischen Ländern und ist daher in der Lage, auch den kulturellen Aspekt von Gesundheit und Krankheit, wie er für westliche Denkungsweise typisch ist, zu berücksichtigen.

Es erscheint daher gerechtfertigt, mögliche Zusammenhänge zwischen der komplexen medizinischen Kur und systemtheoretischen Erkenntnissen zu erörtern. Das Ergebnis dieser Erörterung könnte vielleicht auch einen Beitrag zum besseren Verständnis der Kur und deren Wirkungsgrundlagen in der Medizin leisten.

\section{Systemtheorie in der Medizin \\ $\nabla$}

Wie bereits erwähnt, beruht die klinische Medizin auf naturwissenschaftlichen Denkgrundlagen. Diese Denkgrundlagen weisen einen reduktionistischen Ansatz auf, der sich durch die Forderungen nach einfachen Ursache-/Wirkungsbeziehungen und nach experimentellen Wirkungsnachweisen manifestiert. Nach Ansicht von Ahn et al. [10] ist der Reduktionismus in der klinischen Medizin durch folgende Faktoren gekennzeichnet:

1. dem Fokus auf einzelne, dominante Faktoren

2. der Betonung der Homöostase

3. durch eine ungenaue Risikomodifikation

4. durch additive Behandlungen

Es kann als allgemein bekannt vorausgesetzt werden, dass die Bemühungen der Medizin der Wiederherstellung der Homöostase gelten. Darunter ist die Rückkehr eines Parameters in einen statistisch definierten und statischen Normalbereich zu verstehen. In Übereinstimmung damit steht die Tatsache, dass die naturwissenschaftlich-klinische Medizin sich auf die Annahme gründet, wonach der menschliche Organismus als eine Art von Maschine aufgefasst werden kann. Störungen sind in einer derartigen „Maschine“ immer lokalisiert und spezifisch. Es ist 


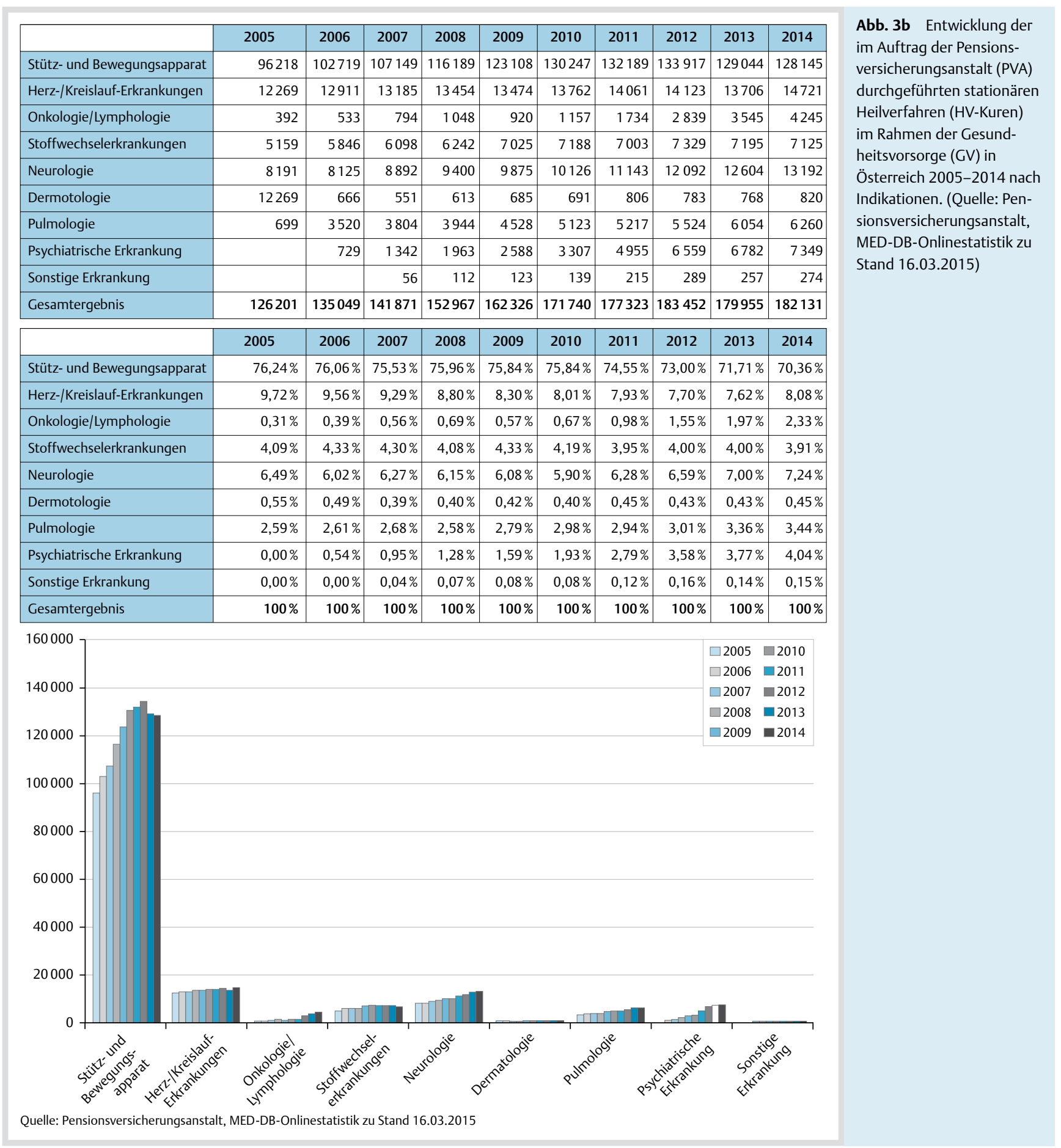

daher eine konsequente Schlussfolgerung, dass auch pathologische Vorgänge lokalisiert und spezifisch sind, wobei davon einzelne Teile oder Systeme des Organismus betroffen sein können. Dies stellt auch die Grundlage der Subspezialisierung in der klinischen Medizin dar. Andererseits muss aber zur Kenntnis genommen werden, dass der Organismus als Ganzes funktioniert und daher auch als Ganzes behandelt werden muss [12].

Erst in der 2. Hälfte des 20. Jahrhunderts entwickelte sich ein Verständnis für die Funktionsweise komplexer Systeme und lieferte so ein Gegenmodell zur Annahme lokalisierter und spezifischer maschineller Störungen. Komplexität kann in verschiedenen Varianten auftreten. Als besonders brauchbar für das Verständnis komplexer biologischer Vorgänge hat sich das Modell von neuronalen Netzwerken erwiesen, welches auch durch parallele Verarbeitungsprozesse charakterisiert ist. Solche Verarbeitungsprozesse treten nicht nur bei neuronalen Vorgängen auf, sondern finden sich auch zwischen den Liganden und Rezeptoren im endokrinen und immunologischen System. Daraus kann abgeleitet werden, dass im Organismus nicht verschiedene voneinander unabhängige Netzwerke vorhanden sind, sondern dass ein ausgedehntes Netzwerk psychoneurologische, immunologische und endokrine Regulationssysteme umfasst [12]. Eine Konsequenz daraus ist, dass ein Fehler innerhalb dieses Netzwerks nicht systemspezifisch ist, sondern sich innerhalb des gesamten Organismus ausbreitet. Es müssen daher 2 unterschiedliche Arten von pathologischen Prozessen unterschieden werden, die 


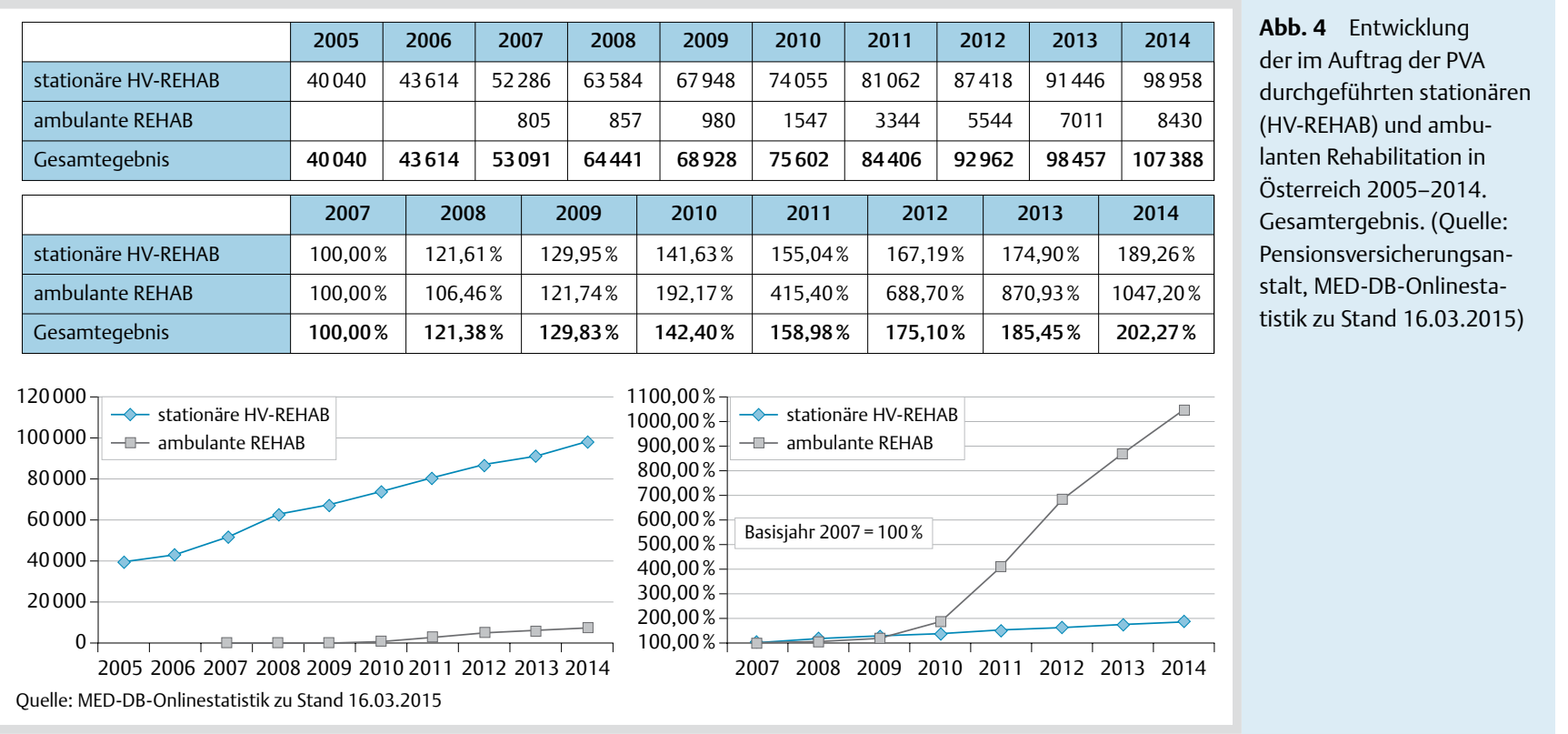

jedoch miteinander in Beziehung stehen: in einem dieser beiden Prozesse können funktionelle Störungen Fehlern spezifischer Strukturen und Systemen zugeschrieben werden, im anderen ist der gesamte Organismus betroffen [12]. Dieser Typ eines pathologischen Prozesses rechtfertigt den Einsatz von therapeutischen Systemen mit verschiedenen Ansatzpunkten, wie die auch für eine komplexe medizinische Kur zutrifft.

Die im vorhergehenden Absatz geäußerten Überlegungen können auch durch weitere Erkenntnisse unterstützt werden. Die Kontrolle bzw. Regulation physiologischer Funktionen wird durch sogenannte negative Feedbacks ausgeübt. Aus konventioneller Sicht werden diese Feedbacks als voneinander unabhängig betrachtet. Die Hypothese eines „intelligenten“ Organismus [12] geht jedoch von der Vorstellung einer Einbettung aller endogenen physiologischen Kontrollsysteme in ein Netzwerk aus. In einem solchen Fall sind die Parameter des Kontrollsystems nicht fixiert, sondern das Netzwerk kann die Parameter des Kontrollsystems in einer Art beeinflussen, die adaptiv für die koordinierte Regulation des Organismus ist. Kontrollsysteme die in ein Netzwerk eingebettet sind haben daher andere Eigenschaften als isolierte Netzwerke. Sie kontrollieren Reaktionsmuster und nicht einzelne Variable und deshalb kann sich das gesamte Kontrollsystem ändern, wenn die verschiedenen Kontrollparameter modifiziert werden. Dies stellt auch die Grundlage für die „Lernfähigkeit" eines komplexen Systems dar, wodurch das System effektiver reagieren kann, als ein sich selbst regulierendes System. Viele so genannte homöostatische Systeme wie z.B. die Thermoregulation oder die Regulation von Hormonkonzentration im Plasma sind ihrer Natur nach nicht homöostatisch sondern homöodynamisch. Sie variieren gesetzmäßig über die Zeit. Um eine homöodynamische Kontrolle zu erzielen, muss das Referenzkriterium einer Kontrollschleife sich über die Zeit verändern und das kann dadurch erreicht werden, dass das Referenzkriterium das Ergebnis des Netzwerks ist. Es existiert eine klare Koordination zwischen verschiedenen Regelkreisen, am auffallendsten ist jedoch jene, die interne und externe Vorgänge bzw. Funktionen kontrolliert. Externe Ereignisse wie z.B. jene die während einer medizinischen Kur einwirken, rufen adaptive physiologische Reaktionen hervor und diese Reaktionen haben psychologische und verhaltensbeeinflussende Folgen. In diesem
Zusammenhang kann erwähnt werden, dass Hildebrandt [18] immer schon auf den Zusammenhang zwischen Kur und physiologische Adaptation hingewiesen hat.

Mit dem Hinweis auf die Bedeutung der physiologischen Adaptation als einer möglichen Grundlage der Wirkung von medizinischen Kuren wird auch der Zeitfaktor angesprochen. Wie dies auch für andere Verfahren mit einem systemischen Ansatz und für jene Verfahren zutrifft, die der Gruppe der Reiz-/Reaktionstherapien zugeordnet werden können, spielt dabei der Zeitfaktor eine wesentliche Rolle. Ein gemeinsames Kennzeichen aller dieser Verfahren ist, dass Serien von im Einzelnen eher subtilen Reizen unterschiedlicher Art eingesetzt werden, wobei die erwünschten therapeutischen Reaktionen keine unmittelbare Folge dieser Reize sind. Durch diese Reize sollen vielmehr physiologische Reaktionen im Organismus von therapeutischer Relevanz ausgelöst werden. Dabei ist zu berücksichtigen, dass komplexe Systeme empfindlich gegenüber den Ausgangsbedingungen sind. Dabei können Reize mit geringer Intensität große Effekte erzielen [9]. Diese Aussage kann zur Erklärung der Tatsache herangezogen werden, dass die im Rahmen einer Kur eingesetzten therapeutischen Anwendungen mit zumeist geringer Reizintensität letztlich doch zu messbaren und nachhaltigen Effekten führen.

Die Entwicklung der therapeutisch relevanten Reaktionen benötigt Zeit. Diese Tatsache wird als sachliche Begründung der Zeitdauer für medizinische Kuren aber auch für andere Verfahren die auf dieser Basis arbeiten, herangezogen. Hayes et al. [19] weisen darauf hin, dass ein dynamisches System als ein Set von Elementen aufgefasst werden kann, die interagieren und sich kontinuierlich über die Zeit entwickeln. Veränderungen können dabei diskontinuierlich auftreten und weisen eine nicht-lineare Charakteristik auf. Es erscheint nicht unberechtigt hierin einen Zusammenhang mit der oft beschriebenen Kurverlaufsdynamik mit mehr oder weniger gesetzlichen Verbesserungen und Verschlechterungen des individuellen Befindens und Gesundheitszustandes zu sehen, die in der Balneologie auch mit dem Begriff Kurkrisen beschrieben werden.

Wie dies von Goldberger [8] betont wird, weisen physiologische Systeme in Gesundheit und Krankheit eine außerordentliche Breite im Hinblick auf das zeitliche Verhalten und von strukturellen Mustern auf, die einem Verständnis Widerstand entge- 


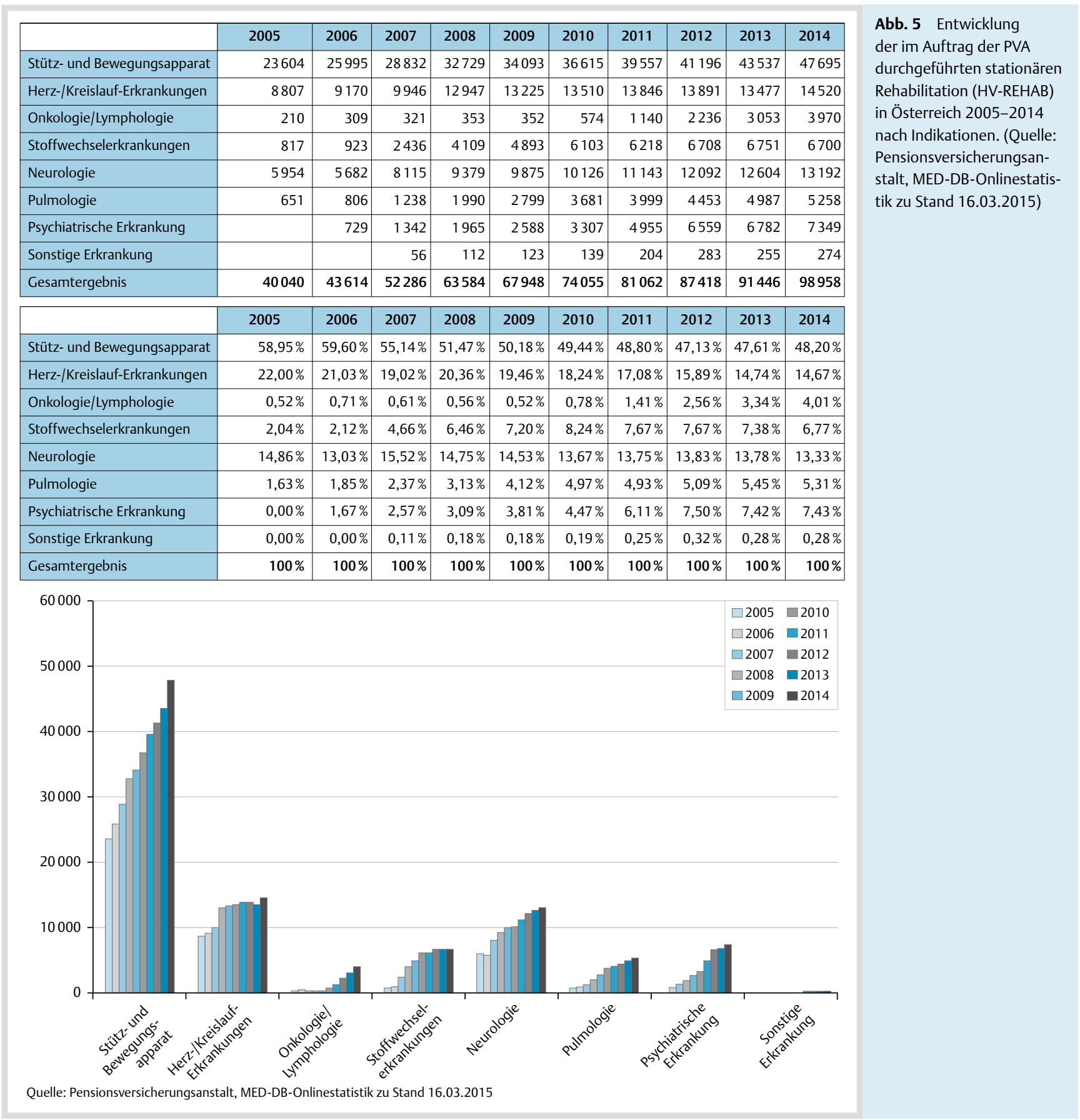

gensetzen, welches auf linearen Denkmustern, reduktionistischen Strategien und der klassischen Homöostase beruhen.

Es kann wohl keinem Zweifel unterliegen, dass es sich beim menschlichen Organismus um ein hochkomplexes System handelt und eine Betrachtungsweise einzelner Bestandteile dieses Systems der Realität nicht gerecht wird. So sprechen Goldberger et al. [17] von einer „verstörenden“ Komplexität als kennzeichnender aber schwer fassbarer Eigenschaft physiologischer Systeme. Diese Komplexität ist zurückzuführen auf die Interaktion von Myriaden struktureller Einheiten und regulatorischer Rückkoppelungsschleifen, die sich über einen weiten Bereich von zeitlichen und räumlichen Skalen erstrecken und den Organismus in die Lage versetzen, auf die alltäglichen Anforderungen zu reagieren und sie zu bewältigen [17].
Komplexität kann aber nicht nur dem gesamten Organismus zugeordnet werden, sondern manifestiert sich bereits auf zellulärer Ebene durch ungefähr 25000 Gene, die für 100000-300000 Proteine kodieren, etwa 1000 Metaboliten und eine nicht definierbare Zahl von verschiedenen Proteinen sowie funktionellen RNA-Molekülen. Die Zahl der zellulären Komponenten, die im intrazellulären Netzwerk fungieren, übersteigt 100000. Die Zahl der funktionell relevanten Interaktionen in diesem Netzwerk ist noch weit höher [13].

Typische Eigenschaften von komplexen, adaptiven und nicht linearen Systemen sind [7]:

1. sie sind ganzheitliche Systeme

2. sie verändern sich über die Zeit

3. sie sind charakterisiert durch Emergenz, Verbindung und wechselseitige Verursachung 


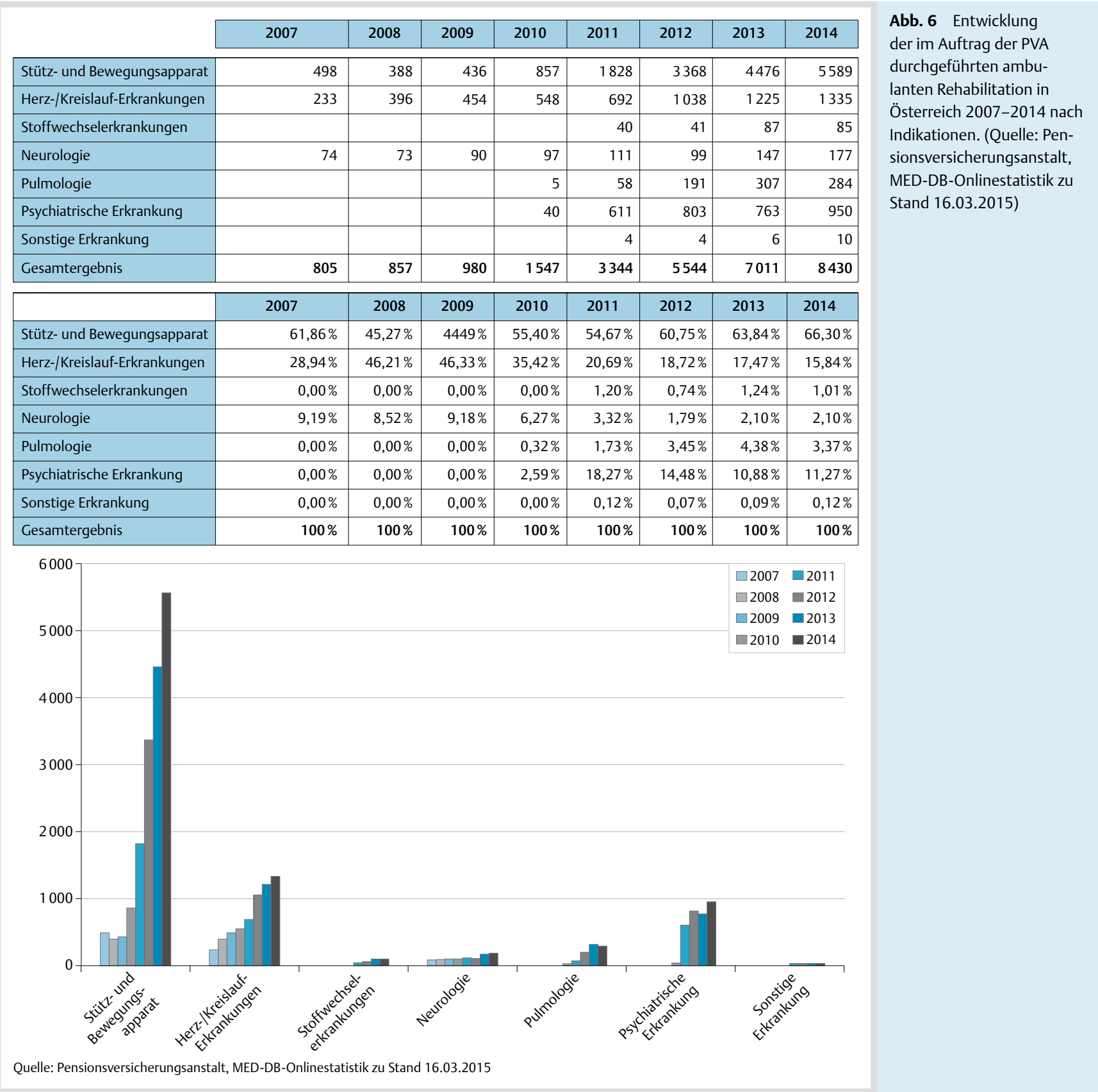

4. Emergenz ist eine Funktion von ganzheitlichen Systemen und nicht durch die Eigenschaften einzelner Teile vorhersagbar

5. Selbstorganisation

6. Verbindung zwischen Stabilität und Flexibilität.

Komplexe adaptive Systeme sind offene Systeme, die sich an die Umwelt adaptieren und mit ihr interagieren. Sie sind ein zusammenhängendes und selbst organisierendes Ganzes mit emergenten Eigenschaften, die größer sind als die Summe der Teile. Die Effekte komplexer medizinischer Kuren beruhen auf einer Mehrzahl von Faktoren, die von außen appliziert werden mit dem Ziel, einen therapeutisch erwünschten Effekt im Organismus zu erzielen. Seit langer Zeit wird dabei zwischen unspezifischen und spezifischen Maßnahmen unterschieden. Den unspezifischen Maßnahmen wird dabei die Funktion zugeschrieben, die Voraussetzung für das Wirksamwerden der spezifischen therapeutischen Maßnahmen zu verbessern. Dies bedeutet aber auch eine Kategorisierung der beiden Gruppen von Maßnah- men, wobei den spezifischen Maßnahmen die eindeutig höhere Bedeutung für den Kurerfolg zugeschrieben wird. Damit in $\mathrm{Zu}-$ sammenhang können auch Bemühungen gesehen werden, kurtherapeutische Maßnahmen auf einzelne definierte und spezifische Anwendungen zu beschränken und alle anderen Faktoren einer komplexen Kur als unwesentlich für den Kureffekt zu beurteilen. In der klinischen Medizin ist es üblich, therapeutische Effekte ausschließlich aus dem Blickwinkel spezifischer und vorhersagbarer Reaktionen in einem System zu beurteilen. Globale Reaktionen werden als systemisches Rauschen betrachtet, dem keine therapeutische Bedeutung zukommt. Systemtheoretische Betrachtungen stellen allerdings das Konzept von spezifischen und unspezifischen Effekten in Frage. In einem komplexen System von Beziehungen mit einer praktisch unbegrenzten Vielzahl von möglichen Feedback-Schleifen und Reaktionen auf eine Reizeinwirkung auf das System, können komplexe adaptive Reaktionen auf verschiedenen Ebenen auftreten, die unvor- 
hersagbar und emergent sind. Es kann daher die Schlussfolgerung gezogen werden, dass innerhalb eines Systems alle Effekte spezifisch sind [7].

Eine Konsequenz aus der Anerkennung der Tatsache, dass komplexe Kuren aus systemtheoretischer Sicht betrachtet und beurteilt werden sollten, hat auch Auswirkungen auf Forschungsdesigns, die geeignet sind, die Effekte solcher gesundheitsförderlicher Verfahren wissenschaftlich zu untersuchen und zu untermauern. Umfassende Angaben zu solchen Forschungsdesigns finden sich u. a. bei Melchart [20] sowie bei Vicent und Furnham [21]. Zu dieser Thematik kann noch hinzugefügt werden, dass die derzeit in der Wissenschaft vorherrschende Sichtweise und die damit zusammenhängenden Untersuchungsmethoden beträchtliche Limitierungen aufweisen, wenn sie auf komplexe und dynamische Prozesse bezüglich Gesundheit und Krankheit beim Einzelnen, in Familien und bei Populationen angewendet werden [7].

Die komplexe Kurmedizin steht einerseits auf dem Boden der Naturwissenschaft, ihre Effekte können aber mit einem reduktionistischen Denkansatz nicht ausreichend beschrieben werden. Andererseits war es die Absicht des vorliegenden Beitrags zu zeigen, dass für die Beurteilung der Wesensmerkmale der komplexen medizinischen Kur systemtheoretische Denkansätze adäquat erscheinen. Die komplexe medizinische Kur könnte daher einen Beitrag zur Integration zweier unterschiedlicher Denkansätze in der Medizin im Interesse der Patienten leisten.

\section{Interessenkonflikt}

$\nabla$

Die Autoren geben an, dass kein Interessenskonflikt besteht.

\section{Literatur}

1 Haber G. Volkswirtschaftliche Effekte des Kurwesens in Österreich. 2014; Endbericht

2 Strausss-Blasche G, Marktl W. Erholungsforschung: der Effekt von Kur- und Erholungsaufenthalten auf die körperliche, emotionale und geistige Befindlichkeit.In: Perspektiven psychologischer Forschung in Österreich. Hsg. Jirasko M, Glück J, Rollett B.WUV Universitätsverlag; 1996: 167-171

3 Strauss-Blasche G, Marktl W. Der Effekt von Kur- und Erholungsaufenthalten auf die Befindlichkeit - Ein Beitrag zur Erholungsforschung.In Kur - Gesundheit - Prävention. Bad Tatzmannsdorfer kurwissenschaftliche Symposiumsreihe Band III. Hsg. Marktl W.Facultas Universitätsverlag; Wien: 1998: 110-114

4 Strauss-Blasche G, Ekemkcioglu C, Klammer N et al. The Change of WellBeing Associated with Spa Therapy. Forsch Komplementärmed Klass Naturheilkd 2000; 7: 269-274
5 Strauss-Blasche G, Muhry F, Lehofer M et al. Time Course of Well-Being after a Three-Week Resort Based Respite from Occupational and Domestric Demands: Carry - Over, Contrast and Situation Effects. J Leisure Research 2004; 36: 293-309

6 Blasche G, Leibetseder V, Marktl W. Association of Spa Therapy With Improvement of Psychological Symptoms of Occupational Burnout: A Pilot Study. Forsch Komplementärmed 2010; 17: 132-37

7 Koithan M, Bell JR, Niemayr K et al. A Complex Systems Science Perspective for Whole Systems of Complementary and Alternative Medicine Research. Forsch Komplementärmed Klass Naturheilk 2012; 19: (Suppl 1): 7-14

8 Goldberger AL. Complex Systems. Proc Am Thorac Surg 2006; 3: 467-471

9 Ahn AC, Nahin RL, Calabrese C et al. Applying Principles from Complex Systems to Studying the Efficacy of CAM Therapies. J Alternative a Complementary Med 2010; 16: 1015-1022

10 Ahn AC, Tewari M, Poon CS et al. The Limits of Reductionism in Medicine: Could Systems Biology Offer an Alternative? PLoSMed 3: e208 doi: $10.1371 /$ journal.pmed.0030208

11 Boon H, McPhershon H, Fleishman S et al. Evaluating Complex Healthcare Systems: A Critique of Four Approaches. eCAM 2007; 4: 279-285 doi:10.1093/ecam/nel079

12 Hyland ME. The Intelligent Body and its Discontents. J. of Health Psychology 2002; 7: 21-32

13 Barabasi A-L, Gulbahce N, Loscalzo J. Network medicine: a network based approach to human disease. Nature Reviews/Genetics 2011; 12: $56-68$

14 Verhoef MJ, Lewith G, Ritenbaugh C et al. Complementary and alternative medicine whole systems research: Beyond identification of inadequacies oft he RCT. Complementary Therapy in Medicine 2005; 13: 206-212

15 Hyland ME. Extended Network Generalized Entanglement Theory: Therapeutic Mechanisms, Emprical Predictions, and Investigations. J Alternative Comp Med 2003; 9: 919-936

16 Barabasi A-L, Gulbahce N, Loscalzo J. Network medicine: a network based approach to human disease. Nature Reviews/Genetics 2011; 12: 56-68

17 Goldberger AL, Peng C-K, Lipsitz LA. What is physiologic complexicity and how does it change with aging and disease? Neurobiology of Aging 2002; 23: 23-26

18 Hildebrandt G. Therapeutische Physiologie.In: Handbuch der Balneologie und medizinischen Klimatologie.Hsg. Chr. Gutenbrunner u. G. Hildebrandt. Springer; 1998: 5-85

19 Hayes AM, Laurenceau J-P, Feldman $G$ et al. Change is not always linear: The study of nonlinear and discontinuous patterns of change in psychotherapy. Clin Psych Rev 2007; 27: 715-723

20 Melchart D.Hsg Naturheilverfahren. Schhattauer Verlag; Stuttgart: 2002

21 Vincent Cha, Furnham A. Complementary Medicine. A Research Perspective. J. Wiley and Sons; West Sussex: 1997 